\title{
The seasonal flu vaccination and uptake among healthcare workers
}

\author{
Emeritus Professor Alan Glasper, from the University of Southampton, discusses the quest by NHS England to \\ encourage nurses and other healthcare workers to have the vaccination
}

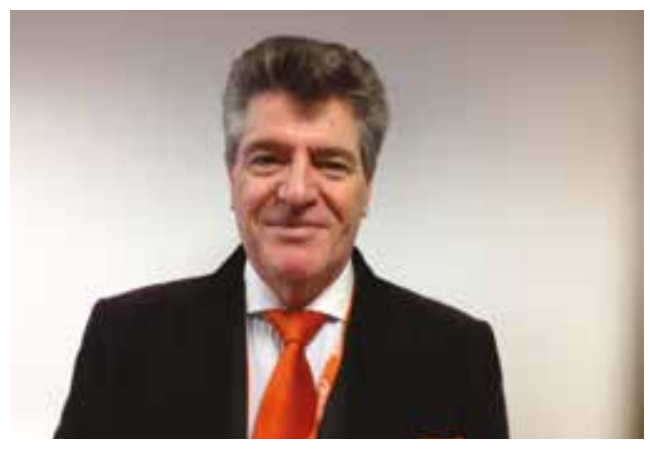

$\mathrm{T}$

here is something dark, sinister and deadly in the neighbourhood, a force so malevolent and contagious that it can silently contaminate a person if it gets within 6 feet of them. This potentially deadly and transmissible force, which takes but few prisoners, is influenza (flu) and it is spread via droplet infection or from contact with contaminated objects such as door handles and then subsequently through touching the eyes, nose or mouth.

Many people see flu as being similar to the common cold, irritating but not especially dangerous. However, this seriously underestimates the power of the virus, which is so much more severe than the common cold and, for many, a fatal illness. In the year following the end of the First World War, in which 17 million people died, the 'Spanish flu' pandemic claimed between 20 and 40 million lives, probably the worst pandemic the world has ever known (Billings, 2007).

In light of the significant threat posed by flu to the population, Public Health England (PHE) has an ongoing strategy for immunising people against infectious disease and this comprehensive plan is set out in Immunisations Against Infectious Disease, the so-called 'Green Book', which has the latest information on vaccines and vaccination procedures, for vaccine-preventable infectious diseases in the UK (PHE, 2014).

In particular, PHE has a specific mandate to tackle seasonal flu on an annual basis, not only to protect the health of the individual but also to ensure that public services such as the NHS and the transport systems are able to function without the devastating loss of the manpower that keeps the wheels of these industries turning. The national flu immunisation programme for 2016-17 was cascaded via a letter from NHS England, PHE and the Department of Health (DH) to all sectors of the NHS, with an emphasis on compliance with flu immunisation uptake by healthcare staff (NHS England et al, 2016).

The DH recognises that frontline NHS vaccination against flu is a major plank in its overall infection-prevention strategy for the service. This is because all frontline health and social care staff have a duty of care to protect their patients and service users from infection. To help improve uptake by staff, NHS Employers has used the 'flu fighter' campaign, which works closely with PHE and the DH to improve levels of flu immunisation among NHS staff (NHS Employers, 2016a).

The uptake of flu immunisation by NHS frontline staff was estimated at 55\% during the period 2013-14, as reported in this column (Glasper, 2014). However, figures released by NHS Employers in November 2016 show that a total of 372339 frontline staff had their flu vaccination in the period from 1 September 2016 to 31 October 2016, equating to $40.4 \%$ of eligible healthcare workers (NHS Employers, 2016b).

\section{Background}

An editorial in The Guardian pointed out that it was a failure by the NHS to provide an effective flu vaccine for the flu season 2015-16 that may have contributed to the largest rise in deaths in England and Wales for 12 years (Batty, 2016). To put this in context, data from the Office for National Statistics (ONS) showed a rise of 28189 deaths in 2015 attributed to flu, the largest year-on-year percentage increase since 1968 (ONS, 2016).
It is not just the deaths caused by flu that is of concern, but the sheer numbers of people who become ill once infected, often for weeks before symptoms abate. During the swine flu epidemic of 2009, for example, a total of 540000 people were infected with the virus (NHS Choices, 2009).

It is interesting to note that in the middle of this season's flu epidemic, Health Secretary Jeremy Hunt launched new plans to reduce infections in the NHS. In November 2016 he announced Government plans to halve the number of Gram-negative bloodstream infections by 2020 . Escherichia coli, which represents $65 \%$ of the Gram-negative infections, caused the deaths of more than 5500 NHS patients last year, which is still significantly less than those killed by flu (DH, 2016).

The Government has decided that, in order to reduce deaths and morbidity from flu during 2016-17, the following groups should be targeted for flu immunisation:

- All children aged 2-7 (but not 8 years or older) on 31 August 2016

- Those aged 6 months to under 65 years in clinical risk groups

- Pregnant women

- Those aged 65 years and over

- Those in long-stay residential care homes

- Carers. (NHS England et al, 2016)

These groups are at particular risk of severe illness if they catch flu-especially the very old and the very young. It is salutary to note that all pregnant women will be offered the vaccination, because flu is the most frequent single cause of death in pregnancy.

However, the flu letter expresses disappointment that vaccination rates dropped for 2-4 year olds in 2015-16, despite the hard work of many health professionals to increase compliance rates. The Government understands that reaching this pre-school group of children continues to be challenging, but still remains important not only for their own protection but also to inhibit the spread 
of flu to other children and to susceptible adults (NHS England et al, 2016).

\section{Promoting NHS staff uptake of flu immunisation}

This year's flu letter confirms that nearly $60 \%$ of NHS staff are not currently immunised against seasonal flu viruses and the Government is determined to increase the uptake of the flu jab among health workers (NHS England et al, 2016). During flu outbreaks it is not uncommon for high levels of sickness among nurses to occur. This puts further strain on nursing staff who remain uninfected and may undermine their ability to maintain high standards of care. The Government is also fearful that were a seasonal flu outbreak to reach pandemic proportions among key nursing personnel, the ability of the NHS to provide a national service could well be compromised.

A number of persuasive strategies are being pursued in the quest to increase flu vaccination uptake among NHS staff. NHS England, for example, is using the Commissioning for Quality and Innovation (CQUIN) scheme. Under this, NHS trusts will be eligible for additional payments if they increase the number of staff vaccinated. However, only those that achieve immunisation levels of $75 \%$ or above will be eligible for the full payment (NHS England, 2016).

The Government believes that flu immunisation should be offered by NHS organisations to all employees directly involved in delivering care. Immunisation against flu should form part of a health employer's policy for the prevention and transmission of flu to protect patients, staff and visitors.

Although the Royal College of Nursing (RCN) rejected the notion of compulsory flu immunisation by an overwhelming majority at the RCN congress in 2012 (RCN, 2012), some organisations that have attempted to follow such a path have seen significant rises in immunisation levels among nursing staff, but with corresponding rises in the number of staff complaints (Isaacs and Leask, 2008).

Despite this, the RCN acknowledges that annual immunisation remains the best way to F protect people against flu, and it has advised its members to be vaccinated, not only to protect themselves but also their patients (RCN, 2016; Donovan, 2016).

The Nursing and Midwifery Council (NMC) Code requires registrants to:
'Take all reasonable personal precautions necessary to avoid any potential health risks to colleagues, people receiving care and the public.'

NMC, 2015: 14

Nurses, midwives and care staff make up the majority of the NHS workforce and together they can mitigate against the spread of flu if they are vaccinated against it. The annual flu letter is counting on the raison d'etre of the nursing profession to 'do the patient no harm' in its quest to motivate staff to comply with the flu vaccine initiative (NHS England et al, 2016).

Frontline healthcare workers such as nursing staff are likely to be exposed to the influenza virus, particularly during winter months, when significant numbers of their patients may be contagious with flu. Worryingly, the flu letter reveals that as many as $25 \%$ of nurses can become infected with the flu virus even during a mild epidemic, a much higher incidence than that seen in the general population. Furthermore, even previously healthy people, but especially the old and the young, can develop severe complications. The flu letter points out that up to one third of deaths during the seasons 2009-10 and 201011 were among patients considered healthy, with many of the cases of severe illness being experienced in people under 65 years of age (PHE, 2016).

\section{Conclusion}

Healthcare workers such as nurses may transmit influenza to patients even if they are showing few symptoms themselves. No nurse wants or seeks to be the harbinger of disease or the cause of a potential death in a frail older person or a sick neonate and it is for this reason that the flu letter is urging all nurses to take advantage of the free flu vaccine. Only through developing a herdimmunity effect among healthcare workers can the NHS reduce the likelihood of the introduction and transmission of the flu virus in healthcare settings. The vaccine has overall efficacy of 59\% against flu in adults aged 18 to 65 years (NHS England, 2015) and its side effects are minor and uncommon. The flu season is a long one and extends well into the new year and all frontline nurses should seek immunisation. BJN

Batty D (2016) Number of deaths in England and Wales hits 12-year high. The Guardian. 7 April. http://tinyurl.com/ gv5moky (accessed 19 January 2017)

\section{KEY POINTS}

- During 2015-16, 28189 deaths were attributed to flu

- The Department of Health recognises that frontline NHS vaccination against flu is a major plank in its overall infectionprevention strategy for the service

- It is not just the deaths caused by flu that is of concern but the sheer numbers of people who become ill once infected

- The Royal College of Nursing has rejected the notion of compulsory flu immunisation for NHS staff

- All frontline nurses should seek flu immunisation. It has an overall efficacy of $59 \%$ and its side effects are minor and uncommon

Billings M (1997) The Influenza Pandemic of 1918. Stanford Education. http://tinyurl.com/lsnvyka (accessed 19 January 2017)

Department of Health (2016) Reducing infections in the NHS. 20 December. http://tinyurl.com/jpugwtx (accessed 19 January 2017)

Donovan H (2016) Protect yourself and your patients this winter. RCN, 3 October. http://tinyurl.com/hr6utex (accessed 20 January 2017)

Glasper A (2014) Should the influenza vaccine be mandatory for frontline staff? Br J Nurs 23(20): 1088-89. http:// dx.doi.org/10.12968/bjon.2014.23.20.1088

Isaacs D, Leask J (2008) Should influenza immunisation be mandatory for healthcare workers? No. BMJ 337: a 2140

NHS England (2016) Flu fighter guidance: CQUIN for flu leads. http://tinyurl.com/j9f7ktk (accessed 24 January 2017)

NHS England, Public Health England, Department of Health (2015) Flu Plan:Winter 2015/16. http://tinyurl.com/ z2689zq (accessed 25 January 2017)

NHS England, Public Health England, Department of Health (2016) The National Flu Immunisation Programme 2016/17. Letter. 26 May. http://tinyurl.com/zcztm2z (accessed 19 January 2017)

NHS Choices (2009) Swine flu deaths examined. http:// tinyurl.com/2bymqdq (accessed 23 January 2017)

NHS Employers (2016a) Flu fighter. http://tinyurl.com/ pa7pqja (accessed 19 January 2017)

NHS Employers (2016b) Latest flu vaccination uptake figures. 17 November. http://tinyurl.com/h67geah (accessed 19 January 2017)

Nursing and Midwifery Council (2015) The Code: Professional Standards of Practice and Behaviour for Nurses and Midwives. http://tinyurl.com/zy7syuo (accessed 24 January 2017)

Office for National Statistics (2016) Excess winter mortality in England and Wales: 2015/16 (provisional) and 2014/15 (final). 23 November. http://tinyurl.com/zotulo5 (accessed 19 January 2017)

Public Health England (2014) Immunisation Against Infectious Disease. Public Health England, London. http://tinyurl. com/nqbpvr5 (accessed 19 January 2017)

Public Health England (2016) Healthcareworker Vaccination: Clinical Evidence (updated August 2016). http://tinyurl. com/h2ew2xo (accessed 24 January 2017)

Royal College of Nursing (2012) Flu jabs for all? RCN congress agenda, 12 . http://tinyurl.com/hrn965w (accessed 24 January 2017)

Royal College of Nursing (2016) Flu vaccinations 2016-2017. Advice guide. http://tinyurl.com/j8oh9mk (accessed 24 January 2017) 